\title{
Die Rache Und das Rätsel der Gabe
}

\author{
AXEL T. PAUL
}

I.

Von Rache ist in jüngster Zeit häufig die Rede, doch nur selten wird sie ausdrücklich zum Thema, vielleicht weil uns zu selbstverständlich erscheint, was mit ihr gemeint ist. Wer hätte nicht schon einmal den Impuls verspürt, sich für eine körperliche oder seelische Verletzung, eine persönliche Kränkung, ein Unrecht, das einem geschehen, einen ungerechtfertigten Angriff, eine Herabsetzung oder eine Beleidigung an ihrem (oder seinem) Urheber rächen zu wollen? Wer ist frei von dem Wunsch, dass denen, die uns Böses tun, Böses widerfahre? Die Rache ist uns vertraut nicht nur als eigenes Gefühl und Handlungsmotiv. Auch aus Geschichte, Literatur und Film schlägt sie uns allenthalben entgegen. Gelten nicht schon oder gerade die Griechen und die Germanen als besonders rachsüchtige Völker? Sind ihre Mythen und Sagen, in denen Götter und Helden sich aus Rache bekriegen, nicht etwa Ausdruck ihrer tatsächlichen Allgegenwart? Was sind die Ilias, das Nibelungenlied oder die Geschichte von Kain und Abel, wenn nicht Rachegeschichten? Gab es im Mittelalter nicht ständig Fehden? Spielt die Rache nicht in den meisten Dramen Shakespeares eine wichtige Rolle? Ist die Folklore von Robin Hood bis Rambo nicht voll von Rächern, die, gleich wie gewalttätig sie sind, unsere Sympathien genießen?

Und doch gilt die Rache als ein Gefühl, das unterdrückt werden muss, als ein rohes und eben nicht zivilisiertes Handeln, dem Einhalt zu bieten ist, wenn ein friedliches Zusammenleben möglich sein soll. Kinder werden dazu ermahnt, sich nicht selbstherrlich und dementsprechend maßlos an ihresgleichen zu rächen, sondern auf die höhere Gerechtigkeit ihrer Eltern zu hören. Eine wesentliche Etappe der Sozialisation ist es, die ungebändigten Triebe einschließlich der Rachsucht an die Kette sowohl eines individuellen Kosten-Nutzen-Kalküls wie vor allem sozialer Normen zu legen. Erst das Verbot, so die nicht bloß psychoanalytische Lehre, im Falle von Konflikten selbst zur Waffe zu greifen, errichtet Kultur. In vielen Western geht es um nichts anderes als darum, dass das Gesetz gegen die Rache durchgesetzt werden muss - und, wenn sie gut sind, 
zeigen sie nicht nur, wie notwendig der Verzicht der Helden auf private >Rechtsprechung< ist, sondern zugleich, wie schwer er ihnen fällt. Dieselbe Spannung zwischen individuell verständlichen Rachegelüsten und dem rechtsstaatlich unabdingbaren Racheverbot lag der öffentlichen Diskussion des Falls Marianne Bachmeier zugrunde, welche Anfang der achtziger Jahre den Mörder ihrer Tochter im Gerichtssaal erschoss. Nicht obwohl, sondern weil Bachmeier >bloß< den gewaltsamen Tod ihrer Tochter rächte, wurde sie selbst (wegen Totschlags) zu sechs Jahren Haft verurteilt. Der Rechtsstaat rächt sich nicht, sondern er bestraft. Nimmt man seine juristische Programmatik beim Wort, fordert ein Rechtsstaat, in dem die Todesstrafe abgeschafft ist, keine Vergeltung, das heißt keine Genugtuung durch eine der Tat proportionale Schädigung des Täters, sondern vielmehr Wiedergutmachung und Rehabilitation. Es geht ihm nicht, wie mutmaßlich der Rache, um den Ausgleich durch eine Erhöhung der Schadensbilanz, sondern um dessen mögliche Begrenzung.

Sowohl die mehr oder weniger unbewusste Konnotation der Rache als eines individuellen Vergeltungsdrangs als auch ihr zumindest vom juristischen Diskurs behaupteter struktureller Gegensatz zum Recht(sstaat) lassen uns erschauern, wenn im gegenwärtigen gewaltsamen Konflikt zwischen Islamismus und westlicher Kultur das Rachemotiv alles andere als fröhliche Urstände feiert. An die Rachespiralen zwischen Israelis und Palästinensern, auch an den Manichäismus Bin Ladens und seines Gegenspielers im Weißen Haus, an ihre Verlautbarungen, dass keine Tat der Gegenseite ungesühnt bleibe, hatte man sich fast schon gewöhnt. Vollends aber mit der am 11. Mai 2004 als Video ins Internet gestellten und damit - wie man mittlerweile weiß - grausam >vorbildlichen` Hinrichtung des US-amerikanischen Zivilisten Nicholas Berg durch eine islamistische Terrorzelle, die als Rache für die Misshandlungen irakischer Kriegsgefangener durch US-Soldaten im Gefängnis von Abu Ghraib deklariert wurde und gerechtfertigt werden sollte, ${ }^{1}$ scheint nicht allein das Rachemotiv, sondern auch eine archaische Rachepraxis ihren Weg zurück in die Moderne gefunden zu haben.

Doch handelte es sich bei der Hinrichtung Bergs wirklich um einen Racheakt? Wenn ja, in welcher Hinsicht? Inwieweit und mit welchen Gründen kann davon die Rede sein, dass die Rache wiederkehrt? Allgemeiner gefragt, sind die Rachegelüste oder ist die Institution der Rache der (Ab-)Grund, über den unsere Zivilisation nur einen dünnen Firniss gelegt hat? Wahrscheinlich sind die Racheimpulse, die jeder von uns kennt oder wenigstens schon einmal verspürt hat - nur so viel zur Psychologie meines Themas, die damit gewiss nicht erschöpft ist -, Klopf-

1 Siehe Dexter Filkins: »Iraq Videotape Shows the Decapitation of an American«, in: New York Times vom 12.05.2004. 
signale aus den Tiefen unserer Psyche, Zeichen einer irreduziblen Spannung zwischen der rohen Natur des Menschen und ihrem kulturellzerbrechlichen Gehäuse. Doch folgt daraus - das ist meine Frage -, dass die Rache das ist oder immer schon war, als was wir sie verstehen?

\section{II.}

Noch einmal: Die diskursive Allgegenwart der Rache steht in deutlichem Gegensatz zur eher dürftigen Behandlung des Themas in den Wissenschaften. ${ }^{2}$ Vermutlich sitzen die Evidenzen zu fest, als dass sie ohne weiteres erschüttert werden könnten. Es gilt als ausgemacht nicht nur, dass die Rache ein dem Menschen (oder sogar höheren Lebewesen) angeborener Impuls ist, ein ihm angetanes Leid durch die Schädigung des mutmaßlichen Täters zu vergelten; sondern auch, dass dieser archaischaggressive Impuls der Strafe vorausliegt, im Zuge der soziokulturellen Evolution aber tabuisiert und durch die Rechtsprechung ersetzt worden ist. Vorgeführt und bewiesen wird die Dysfunktionalität und Unhaltbarkeit der primitiven Rache in der Regel durch den Verweis auf die Institution der Blutrache, die vorschreibe, dass ein schweres Verbrechen und vor allem jeder Mord nur durch einen weiteren Mord gesühnt werden könne.

Die Rache stellt also einen unendlichen, endlosen Prozeß dar. Wann immer sie an einem beliebigen Punkt innerhalb einer Gesellschaft auftaucht, neigt sie dazu, sich auszubreiten und die gesamte Gesellschaft zu erfassen. Sie droht eine wahre Kettenreaktion auszulösen [...]. Mit der Häufung der Vergeltungsmaßnahmen wird die Existenz der Gesellschaft insgesamt aufs Spiel gesetzt. $^{3}$

Gleichwohl, die Blutrache hat sich, abgesehen von vereinzelten zeitgenössischen Beispielen aus dem Balkan, dem Kaukasus und der Unterwelt

2 Zum besten, was in jüngerer Zeit über die Rache geschrieben worden ist, gehört Raymond Verdier: "Le Système vindicatoire. Esquisse théorique «, in: ders. (Hg.), La Vengeance. Etudes d'ethnologie, d'histoire et de philosophie, Bd. 1: Vengeance et pouvoir dans quelques sociétés extra-occidentales, Paris 1981, S. 11-42; instruktiv auch Jonathan Rieder: "The Social Organization of Vengeance «, in: Donald Black (Hg.), Toward a General Theory of Social Control, Bd. 1: Fundamentals, Orlando 1984, S. 131162; herausragend aus der älteren Literatur Hans Kelsen: Vergeltung und Kausalität (engl. Orig. 1943), Wien 1982, §§ 17-24; einen breiten Überblick über die Verarbeitung des Themas in Literatur, Hochreligionen, Psychologie und Rechtsgeschichte gibt Susan Jacoby: Wild Justice. The Evolution of Revenge, New York 1983.

3 René Girard: Das Heilige und die Gewalt, Zürich 1987, S. 28. 
der Großstädte unseres Globus, nicht halten können, die Gesellschaft hingegen sehr wohl.

René Girard zufolge, von dem das vorstehende Zitat stammt, war es die Erfindung und Institutionalisierung des Opferrituals, das im Kern nicht etwa eine Kommunikation der Menschen mit ihren Göttern, sondern vielmehr die Um- und Ableitung der »wesenhafte[n] Gewalt « ${ }^{4}$ primitiver Gesellschaften respektive von Gesellschaft überhaupt auf ein unschuldiges und deswegen die Rachespirale unterbindendes Opfer darstelle. Der Opfer- beziehungsweise Sündenbockmechanismus ist zweifellos ein probates und wahrscheinlich sogar universales Mittel, Rachezirkeln zu entkommen. Sehr viel näher jedoch hätte der empirische Hinweis gelegen, dass die Blutrache alles andere als eine universale Institution war und selbst dort, wo es sie gab, sehr viel seltener und maßvoller praktiziert wurde als angenommen. ${ }^{5} \mathrm{Zu}$ erklären ist also weniger, wie Gesellschaften aus Rachespiralen zu entfliehen vermochten, als vielmehr, was sie allererst zur Blutrache trieb.

Bereits eine Auflistung der Gesellschaften, für welche die Blutrache zweifelsfrei dokumentiert ist, legt eine Antwort nahe. Praktiziert wurde die Blutrache - ohne Anspruch auf Vollständigkeit - im Mittelmeerraum auf einigen der größeren Inseln (Korsika, Sardinien, Sizilien) sowie im gebirgigen Hinterland (Balkan, Rif), im Kaukasus, im schottischen Hochland, auf Island, bei den Eskimo, auf Neuguinea und den Philippinen (Luzon), im südostasiatischen Bergland, im Sahel- und AmazonasGebiet. Schon die geographische Verteilung der Fälle, aber auch ihre zeitliche Distanz zueinander schließen aus, dass die Blutrache um die Welt diffundiert sein könnte. Demnach muss es strukturelle Gründe geben, die ihr Auftreten erklären. Tatsächlich handelt es sich allen beachtlichen kulturellen Differenzen zum Trotz stets um segmentäre Gesellschaften, die unwegsame, relativ unwirtliche Gebiete besiedeln, in welchen staatliche Herrschaftsstrukturen sich entweder gar nicht oder nur rudimentär entwickeln konnten. Offensichtlich erfüllt die Blutrache, auch wenn sie weder eine kulturgeschichtlich universale Institution darstellt, noch von allen segmentären Gesellschaften ausgeübt wurde, einen Zweck, der unabhängig von der konkreten Gestalt, den Werten und Ideen der jeweiligen Gesellschaft, in denen man sie praktizierte, verstanden werden kann.

Segmentäre Gesellschaften sind Gesellschaften ohne Staat, ohne politisch zentrale, über eine legitime Zwangsgewalt verfügende Institutio-

4 Ebd., S. 49.

5 Siehe E. Adamson Hoebel: »Feud: Concept, Reality and Method in the Study of Primitive Law «, in: Akshayakumar R. Desa (Hg.), Essays on Modernization of Underdeveloped Societies, Bd. 1, Atlantic Highlands, N.J. 1976, S. 500-513. 
nen. Es mag Anführer geben, ihr Machtbereich ist dann jedoch auf bestimmte Aufgaben wie die Organisation der Jagd oder die Kriegsführung gegen andere Gruppen beschränkt. Segmentäre Gesellschaften werden aus diesem Grund auch als akephale, das heißt 〉kopflose` Gesellschaften bezeichnet. Ihre grundlegende Einheit ist das Segment. Segmente sind, abgesehen davon, dass ihre Frauen ausheiraten und sie selbst folglich auf die Frauen anderer Segmente angewiesen sind, materiell in sich (überlebens-)fähige Gruppen, die zusammen mit anderen gleicher Sprache, Religion und »Ökologie« eine Gesellschaft bilden. Sie bewirtschaften denselben geographischen Lebensraum und gehen im Prinzip denselben Tätigkeiten nach: dem Jagen und Sammeln, unter Umständen der Viehzucht und dem Gartenbau. Der Handel kommt gelegentlich vor, spielt aber keine reproduktive Rolle. Die Arbeitsteilung wie die soziale Differenzierung sind gering entwickelt. Wo überhaupt vorhanden, bilden Sesshaftigkeit und Ackerbau meist schon den Übergang zu Gesellschaften eines sozialstrukturell anderen (stratifizierten) Typs. Fällt ein Segment aus, steht damit die Existenz der Gesellschaft als Ganzer nicht auf dem Spiel. Gebildet werden die einzelnen Segmente aus Clans oder Familien; häufig sind Familie oder Clan und Segment deckungsgleich. Daher, das heißt als Folge des auch hier grundlegenden Inzestverbots, ihre Abhängigkeit voneinander, aber eben auch ihr Zusammenhalt als Geber und Nehmer heiratsfähiger Frauen.

Als die Wissenschaft sich um 1900 für sie zu interessieren begann, galten segmentäre Gesellschaften - in befremdlichem Gegensatz übrigens zur mutmaßlichen Allgegenwart der Blutrache ${ }^{6}-$ als außerordentlich konfliktarm und konsensual integriert. Nachhaltig erschüttert wurde diese Sicht der Dinge mit Bronislaw Malinowskis Crime and Custom in Savage Society. ${ }^{7}$ Allerdings zeigte dieser nicht nur, dass einfache Gesellschaften faktisch etliche Normverstöße und gewaltsame innergesellschaftliche Konflikte kennen und ahnden, sondern auch, dass ihnen mit dem Reziprozitätsbeziehungsweise Vergeltungsprinzip ein wirkungsvoller >privatrechtlicher Regelungs- und Konfliktschlichtungsmechanismus zur Verfügung steht. Auch ohne ein gesetztes, kodifiziertes und von mehr oder weniger neutralen Instanzen überwachtes und angewandtes Recht gibt es in Gesellschaften ohne Staat und Schrift eine auf die Überlieferung gestützte Rechtsmoral, gibt es Rechtsprechung und Rechtsvollzug. Jede Gesellschaft verfügt

6 Vgl. Sebald R. Steinmetz: Ethnologische Studien zur ersten Entwicklung der Strafe. Nebst einer psychologischen Abhandlung über Grausamkeit und Rachsucht, 2 Bde., 2. Aufl., Groningen 1928; Richard Thurnwald: »Art. 'Blutrache « in: Reallexikon der Vorgeschichte, Bd. 2, Berlin 1925, S. $30-41$.

7 Bronislaw Malinowski: Crime and Custom in Savage Society, London 1926. 
über diesseitige Normen, über Ge- und Verbote, die im Fall ihrer Verletzung sanktioniert werden.

Das Vergeltungsprinzip nun ist zugleich Grund wie Maßstab der Strafe. Grund ist es, insofern es vorschreibt, dass ein Vergehen so gut wie eine Gabe erwidert werden muss - ich komme darauf zurück -, Maßstab, insofern es für bestimmte Vergehen bestimmte Sanktionen verlangt. Die Regel, dass ein Vergehen sanktioniert werden muss, bedeutet freilich nicht, dass stets Gleiches mit Gleichem vergolten würde. Eine solche Auffassung setzte nämlich voraus, dass es einen allgemeinen, Tat und Vergeltung gemeinsamen (Wert-)Maßstab gibt. Das nicht nur biblische »Aug' um Auge, Zahn um Zahn« belegt zwar, dass ein zugefügter Schaden, wenn möglich, durch einen spiegelbildlichen Schaden vergolten werden soll; in vielen Fällen jedoch ist eine derartige spiegelbildliche Schädigung ausgeschlossen - dann etwa, wenn (weiblicher) Ehebruch mit dem Tode bestraft wird. Der Grundsatz der Vergeltung schließt die (unterstellte) Identität der beiden Taten nicht aus, entscheidend aber für den Anspruch auf sowie das Ausmaß der Vergeltung ist, dass zwischen erster Tat und Vergeltung überhaupt eine (angenommene) Wertbeziehung besteht. Was uns auf der einen Seite als maßlos erscheint wie die Steinigung einer Ehebrecherin und auf der anderen deshalb empört, weil alle Strafe fehlt, etwa wenn ein Vater seinen ungehörigen Sohn erschlägt, ist nicht an sich ungerecht und vor allem kein Ausweis von Willkür, wenn und insofern die Rechtstradition einer Gesellschaft erlaubte von unerlaubten Taten scheidet, für letztere gewisse Sanktionen bereithält und damit der möglichen Maßlosigkeit der Geschädigten einen Riegel vorschiebt. Schon als bloße Regel und nicht erst als lex talionis bezwingt das Reziprozitätsprinzip den Rachedurst und transformiert ihn in einen Rechtsanspruch. ${ }^{8}$

Im Unterschied zu dem, was eine vorschnelle Identifikation von Vergeltung und >wilder Rache` suggeriert, ist das Prinzip der Vergeltung kein Freibrief zur Barbarei, sondern vielmehr eine Regel der Mäßigung. ${ }^{9}$ Dieser Grundsatz regiert die Blutrache so gut wie jede andere Form von Sanktion. Zunächst nämlich muss festgestellt und öffentlich anerkannt werden, dass eine Tat zur Blutrache berechtigt. Richtig ist zwar, dass die Intention des Täters unter Umständen dann keine Rolle mehr spielt, wenn der zugefügte Schaden ein bestimmtes Maß überschreitet, falsch jedoch

8 Auf einem anderen Blatt steht, dass das Recht auf Vergeltung angerufen werden kann, um es für wahrhaft private Zwecke zu missbrauchen, dass das Fehlen eines kodifizierten Rechts der strategischen Manipulation der Rechtstradition großen Spielraum lässt. Doch auch die Differenz von Rechtsideal und Rechtswirklichkeit, die Instrumentalisierung des Rechts durch die Macht, ist kein Privileg akephaler Gesellschaften.

9 »Ultimately, a theory of vengeance is at one and the same time an explanation of the conditions for its renunciation. « (J. Rieder: The Social Organization of Vengeance, S. 158.) 
ist die verbreitete Annahme, die streitenden Parteien wären der Unterscheidung und differenzierten Bewertung von Vorsatz und Versehen nicht fähig. Weiter ist es nicht jedem Mitglied der geschädigten Gruppe erlaubt, seinerseits einem beliebigen Angehörigen der Tätergruppe irgendeinen Schaden zuzufügen. Vielmehr ist innerhalb eines gewissen Rahmens festgelegt, wer sich auf welche Weise an wem zu rächen hat. Und hier ist es insbesondere die interne Differenzierung der Familienbeziehungsweise Clanstrukturen, welche eine präzise Dosierung der Rache ermöglicht. ${ }^{10}$ So kann die Vorschrift zum Beispiel lauten, dass der Sohn die Ermordung seines Vaters legitimerweise durch die Ermordung des Täters oder eines seiner Brüder rächen darf. Damit wären Gerechtigkeit und sozialer Friede wiederhergestellt. Tötet der Rächer hingegen einen Sohn des Mörders, oder rächt sich nicht der Sohn, sondern ein entfernterer Verwandter des ursprünglichen Opfers, kann es in der Tat sein, dass die Rache als überzogen angesehen wird und zur Vergeltung der Vergeltung einlädt, wenn nicht gar auf sie verpflichtet. Rachespiralen haben dort, wo sie unter den genannten Bedingungen vorkommen, also weder abgründigen Hass noch unstillbaren Rachedurst, sondern eine (unter Umständen auch kalkuliert) gestörte Symmetrie zur Grundlage. ${ }^{11}$ Umgekehrt scheinen eine gewisse Gruppengröße und verwandtschaftliche Binnendifferenzierung das Umschlagen >gerechter Blutrache in maßlose Fehden strukturell zu hemmen.

Stets aber ist es die öffentliche Meinung, welche in letzter Instanz über die Legitimität der Blutrache entscheidet, welche Konflikte eskalieren lässt oder sie unterbricht (zum Beispiel indem sie in Gestalt eines Ältestenrats ein Ordal anordnet, einen Schlichter benennt, ein Opfer inszeniert oder auch einen externen Killer anheuert, um die Streithähne aus dem Verkehr zu ziehen), welche die Blutrache gutheißt oder erzwingt. Ja, es kann sein, dass der Schaden, welcher einer Gruppe oder einem Individuum in Form öffentlicher Missachtung oder gar der `Exkommunikation< daraus erwächst, sich der Rachepflicht zu entziehen, schwerer wiegt als das Unrecht, das sie begründet. Nicht immer interveniert die öffentliche Meinung schlichtend; manchmal ist sie es vielmehr, die >freie Geister< zurückzwingt in die Bahnen der Tradition. Im guten wie im schlechten jedoch ist die Rache in segmentären Gesellschaften nicht Sache eines einzelnen. Nicht er ist es, der rächt, der rächen kann oder muss, der seiner Gefühle nicht Herr wird und von keiner höheren Gerechtigkeit weiß, der seinen Schmerz nicht anders stillen und seine Trauer nicht anders

10 Diese Beobachtung bei Roger V. Gould: »Revenge as Sanction and Solidarity Display: An Analysis of Vendettas in Nineteenth-Century Corsica«, in: American Sociological Review 65 (2000), S. 682-704, hier S. 699-702.

11 Siehe Max Scheler: Das Ressentiment im Aufbau der Moralen, Frankfurt/Main 1978, S. 4-10. 
bewältigen kann als durch den Stoß ins Herz seiner Feinde. All das mag es geben und wird es ihm fraglos erleichtern, seine Rolle als Rächer zu spielen, zuerst und vor allem aber ist die traditionelle Rache ein sozialer Tatbestand, eine Beziehung, die zwischen Kollektiven und nicht zwischen Individuen gelebt wird.

Neben ihrer soziostrukturell verständlichen und faktisch, wenn auch nicht stets, so doch häufig konfliktregulierenden Funktion gibt es noch einen weiteren grundlegenden Aspekt, welcher die Annahme Girards, primitive Rachegesellschaften, die das Opfer und seine juridischen Substitute nicht kennen, segelten hart an der Grenze der Selbstauslöschung, ins Reich der Fabel verweist und darüber hinaus die These, dass es sich bei der Rache um eine erste und rohe Form der Strafe handelt, ins rechte Licht rückt. Denn auch wenn die Vergehen, die zu ahnden in akephalen Gesellschaften der Rache überlassen wird, in Gesellschaften, welche eine politische Zentralinstanz ausbilden, zumindest idealiter von dieser beziehungsweise einer über den Kontrahenten stehenden Judikative verfolgt und gerichtet werden, steht die Rache grundsätzlich nicht im Gegensatz zur Strafe, sondern neben ihr. ${ }^{12}$ Die Kehrseite der Tatsache, dass die Rache zwischen und an Gruppen verübt wird, besteht darin, dass sie innerhalb der rachefähigen und -berechtigten Kollektive untersagt ist. »People whose face is known should not be eaten. ${ }^{13}$ Die für die Blutrache konstitutive Solidarität der Rachegenossen, der Umstand also, dass als Täter wie Opfer ein im heutigen strafrechtlichen Sinne rechtloser oder unschuldiger, auf jeden Fall unbeteiligter Dritter fungiert, hat zur Voraussetzung, dass scheinbar dieselben Vergehen, die auf der einen Seite, nach außen hin, einen Racheanspruch begründen, auf der anderen Seite, nach innen hin, ungerächt bleiben. Ungerächt, deswegen aber nicht - und das ist die Pointe - ungestraft. Selbstverständlich ist ein Mord auch und gerade innerhalb einer Familie oder eines Clans ein Mord, der sanktioniert wird, und kein lässlicher Lapsus. Anders als die heutigen Verfechter der Todesstrafe es gerne hätten, ist es allerdings eher selten, dass auf einen gruppen-, familien- oder claninternen Mord die Todesstrafe steht. ${ }^{14}$ Dort, wo es sie gibt, berechtigt die Hinrichtung des Mörders, der selbstverständlich ermittelt wird und als einzelner für seine Tat haftet - soviel zur vermeintlichen Unfähigkeit primitiver Gesellschaften, Schuld individuell

12 Vgl. R. Verdier: Le Système vindicatoire, S. 14, 22-24.

13 Klaus-Friedrich Koch: War and Peace in Jalémó. The Management of Conflict in Highland New Guinea, Cambridge, Mass. 1974, S. 80.

14 Die Nuer zum Beispiel definieren einen Stamm geradezu über die Möglichkeit eines friedlichen Ausgleichs bei Tötungsdelikten. Siehe Edward E. Evans-Pritchard: »The Nuer of the Southern Sudan«, in: Meyer Fortes/Edward E. Evans-Pritchard (Hg.), African Political Systems, London 1940, S. 272-296, hier S. 278. 
zu attribuieren -, jedoch niemanden - wen auch? -, sich an dem oder den Henkern zu rächen. Dies würde die Einheit der Gruppe sehr viel stärker bedrohen als die Kollektivhaftung, die auf sie fällt, wenn aus ihr heraus oder gegen sie gehandelt wird. Vielleicht lässt sich sogar sagen, dass die äußere Pflicht zur Rache und das innere Verbot von Rache nicht bloß strukturell korrespondieren, sondern dass die Praxis und der Diskurs der Rache die Kohäsion der Gruppe, wenn schon nicht erzeugen, so doch öffentlich dokumentieren und verstärken. Ist schon die Rede von wilden Rachekriegen zwischen aufs Blut verfeindeten Gruppen mit äußerster Vorsicht zu genießen, so gilt erst recht in Hinblick auf die kollektiven Akteure der Rache selbst, dass sie den sozialen Zusammenhalt nicht etwa aufs Spiel setzt, sondern vielmehr integriert.

Eine weitere, wenigstens idealtypische, Unterscheidung ist daher angezeigt. So wie die Rache sich niemals gegen die eigene Gruppe richtet, so wenig ist sie Krieg gegen andere Gruppen. Gerächt wird innerhalb einer Gesellschaft, zwischen den Segmenten, aus denen sie besteht. Gerade die zum Teil akribische Verregelung der Rache, die Tatsache, dass ihrer Eskalation zu Rachespiralen hohe Hürden vorgebaut sind, dass man dort, wo es zur Blutrache kommt, immer auch Verfahren kennt, die ihr Einhalt gebieten, beweist ja gerade, dass die Racheakteure ein und demselben sozialen und kulturellen Zusammenhang angehören. Außerhalb der Gesellschaft steht, wer den eigenen Regeln der Rache nicht unterliegt. Kriege zielen auf die Schwächung, Vertreibung, Unterwerfung oder - auch in vormodernen Zeiten ${ }^{15}$ - Auslöschung konkurrierender Kollektive. Die Anerkennung anderer Gesellschaften respektive anderer politischer Verbände als anderer und doch gleicher, die Respektierung von kulturellen und politischen Differenzen und die entsprechende Bereitschaft, neben Assimilation und Negation dritte Wege sowohl zu sehen als auch zu gehen, ist eine menschheitsgeschichtlich späte und, wie der gegenwärtige Kampf der Kulturen zeigt, hochgradig prekäre zivilisatorische Errungenschaft. Das bedeutet natürlich nicht, dass totale Kriege die vormoderne Regel und in der Moderne lediglich Ausnahmen wären. Dazu fehlten traditionalen Gesellschaften nicht bloß die militärischen Mittel, sondern vor allem das ideologische Rüstzeug, das die Exklusion anderer bis hin zu ihrer Vernichtung nicht allein als unter Umständen praktisch geboten erscheinen ließ, sondern als im Namen der Einen Wahrheit zwingend aufgab. Zudem heißt das Fehlen von Völker- und Kriegsrecht nicht, dass segmentäre Gesellschaften über keinerlei Möglichkeiten verfügt hätten, Kriege anders als militärisch zu entscheiden. Kapitulationen und förmli-

15 Siehe Kurt Jonassohn: Genocide and Gross Human Rights Violations in Comparative Perspective, New Brunswick, N.J. 1998, Kap. 17. 
che Friedensschlüsse waren vielmehr die Regel. ${ }^{16}$ Was ihre Kriege jedoch von der Rache abhebt, ist, dass diese überhaupt nur zwischen Gleichen möglich ist und nicht auf die Vernichtung des Gegners, sondern auf die Wiederherstellung einer Art von Gleichgewicht zwischen den Gruppen zielt. Mit anderen Worten, die Rache selbst ist ein Anerkennungsverhältnis.

\section{III.}

Verschlossen bleiben muss diese Einsicht freilich allen, welche die Rache wie auch, genereller, die Gabe als einen Tausch von Leistungen oder gar Gegenständen verstehen wollen. ${ }^{17}$ Im Falle der Gabe mag eine derartige Auffassung auf den ersten Blick immerhin nahe liegen. Getauscht würden dann Gaben und nicht Waren, entweder weil den Tauschpartnern die kognitiven und technischen Kompetenzen fehlten, zwischen Gabe und Gegengabe eine mehr oder weniger feste Wertrelation zu etablieren, oder aber weil normative und dingliche Gründe einem rationalen Tausch entgegenstünden - sei es, dass der Austausch bestimmter Güter und Leistungen mit einem Verbot belegt ist, sei es, dass die Natur der Dinge (etwa der Liebe oder der Achtung) ihrer Konvertibilität Schranken setzt. Der Gabentausch wäre dann ein Derivat, eine defiziente Form des eigentlichen oder rationalen Tauschs, dessen Grund oder Motiv das vermeintlich natürliche Interesse der Tauscher an der Verbesserung ihrer individuellen Kosten-Nutzen-Bilanz darstellen soll. In der Tat macht die moderne Theorie der rationalen Wahl aus der für die Soziologie konstitutiven Not, soziales Handeln und sozialen Zusammenhalt zu erklären, eine Tugend, indem sie den kalkulierten Tausch zweier Individuen zum heuristischen Schlüssel aller sozialen Phänomene überhaupt erklärt. Jede Interaktion, aber auch jede soziale Beziehung, jedes soziale Gebilde mehr oder weniger dauerhafter Natur, ist in dieser Perspektive ein vernünftiger Tausch, wenn und insofern die Tätigkeit eines Individuums zugleich den Anreiz und die Belohnung der Tätigkeit eines anderen Individuums ausmacht. Was und selbst ob freiwillig getauscht wird, ist dabei eine zweitrangige Frage, denn schließlich lässt sich noch die Unterwerfung unter einen überlegenen Machthaber als ein Tausch von Gehorsam gegen körperliche Unversehrtheit interpretieren. Rational daran ist, dass dem Unterlegenen sein Leben mehr bedeutet als die frühere Freiheit, während der Überlegene die Kosten zumindest einer ersten, überzeugenden De-

16 Vgl. Paul Bohannan (Hg.): Law and Warfare. Studies in the Anthropology of Conflict, Austin, London 1967.

17 So z.B. Hartmut Esser: Soziologie. Spezielle Grundlagen, Bd. 3: Soziales Handeln, Frankfurt/Main, New York 2000, S. 353-364. 
monstration seiner Macht nicht scheut, um in den Genuss des Gehorsams zu kommen. Handlungstheoretisch wird damit aus Max Webers am Idealtypus der Zweckrationalität orientierter Motivsuche ein Monismus des Krämergeists. Einer solchen Tauschtheorie ist nichts Menschliches fremd. Nichts kann vor ihrem scharfen und kalten, von keiner Gefühlsduselei angekränkelten, aller Ideologie außer der des Marktes abholden Blick bestehen. Außer - zumindest - der Rache.

Rache, aufgefasst als das Verhalten eines Individuums, jemanden, den dieses für die Schädigung seiner verantwortlich macht, im Gegenzug zu schädigen, verbunden freilich mit Kosten und nicht unerheblichen Risiken, ist einer strikten rational-choice-theoretischen Deutung nicht zugänglich. Denn was ist, worin liegt der Nutzen der Rache für den Rächer? Eventuell in der Befriedigung, die es bringt, jemanden leiden zu lassen, der uns leiden gemacht hat. Rational aber wäre nur ein Verhalten, dessen Aufwand, den Täter zu stellen und ihn zu >bestrafen`, sowie dessen (kostenträchtiges) Risiko, selbst erneut Schaden zu nehmen, nicht nur geringer zu veranschlagen wäre als die erhoffte Schadenfreude, sondern das im Vergleich zu alternativen >Investitionsmöglichkeiten`zudem den höchsten Ertrag abwürfe. Gewiss ließe sich argumentieren, dass die Rache da, wo gerächt wird, für den Rächer süßer sein muss als das, was sie ihn kostet, dass das Racheverhalten mithin, insofern es sich rational erklären lässt, auch rational sein muss. Will man einer solchen Tautologie jedoch entkommen, wird man Gründe ins Feld führen müssen, welche die Rache, wenn schon nicht objektiv, so doch nachvollziehbar rationalisieren.

Wenigstens drei Argumente sind denkbar. ${ }^{18}$ Erstens könnte es sein, dass die Rationalität der Rache nicht in ihrem Vollzug, sondern in ihrer abschreckenden Wirkung liegt. Zweifellos ist die Institution der Rache ein funktionales Äquivalent für kodifiziertes Recht und organisierte Gerichte. Äußerst fraglich aber ist, ob den Mitgliedern akephaler Gesellschaften die bewusste Kalkulation ihrer pazifizierenden Effekte unterstellt werden kann. Zudem wäre die Rachedrohung glaubhaft nur unter der (empirisch falschen) Annahme einer ungezügelten Eskalation von Rachespiralen. Der Preis, der zu zahlen wäre, sollte die Abschreckung versagen, wäre unter dieser Voraussetzung die Selbstzerstörung der Gesellschaft. Ein wenig triftiges und gewiss nicht rationales Szenario. Wie aber - so ein zweiter Anlauf, die Rache srational zu begründen - steht es um das Verhalten einzelner, die sich trotz oder gerade wegen der verbreiteten und berechtigten Angst vor Rachezirkeln als rachsüchtig ausgeben? Wäre es für einzelne nicht durchaus rational, Rachebereitschaft zu simu-

18 Siehe Jon Elster: »Norms of Revenge«, in: Ethics 100 (1990), S. 862-885, hier S. 872-876. 
lieren und sich dadurch Vorteile zu erzwingen? Gewiss, allerdings wäre eine derartige Simulation plausibel nur dann, wenn es wirklich rachebereite Individuen gäbe, die zu provozieren man lieber vermiede. Damit aber wäre bloß die Existenz irrationaler Personen und gerade nicht die Rationalität der Rache bewiesen. Drittens endlich ist es in der Tat möglich, dass die Kosten, die anfallen, einer allgemeinen Rachenorm nicht zu genügen, höher sind als die, ihr Folge zu leisten. Allerdings setzt dieses Argument voraus, dass es Dritte gibt, welche die Einhaltung der fraglichen Rachenorm überwachen, deren Bruch sanktionieren und die Kosten dieser Sanktion für geringer erachten als den Schaden, der aus dem Bruch der Norm resultiert. Spätestens wenn man sich klarmacht, dass es die faktische Intervention dieser Dritten überwachende >Vierte muss, die ihr Einschreiten wiederum höher bewerten als das Versäumnis der Hüter der eigentlichen Rachenorm - und so weiter, und so fort -, wird das Argument brüchig - es sei denn - dies aber wäre ein Sprung heraus aus der Theorie -, die Norm würde aus anderen als individuellzweckrationalen Gründen befolgt.

Genau dies ist nun aber der Fall. Die Rache, nicht anders als der Gabentausch, geht nicht auf in den Kosten-Nutzen-Kalkülen Einzelner. Ein Tausch ist nicht einfach ein Tausch. Sehr wohl ist darauf zu achten, was und warum jeweils getauscht wird, nicht aber, um die Beschränkungen eines >einfachen Tauschs zu den Bedingungen des Gabentauschs zu verklären, sondern um sich der Einsicht in die grundsätzliche Differenz dieser beiden Typen des Tauschs nicht zu verschließen. Die Rache ist nicht nur ein Phänomen, an dessen Erklärung die Rational-Choice-Theorie scheitert, sondern ein trefflicher Gegenstand, das Wesen oder, um es mit einem Titel Maurice Godeliers zu sagen, das »Rätsel der Gabe« zu lüften. ${ }^{19}$

Der Gabentausch ist - anders als die (Blut-)Rache - ein kulturell universelles Phänomen. In unseren zeitgenössischen Gesellschaften spielt er vordergründig eine nur noch marginale Rolle. Das Standardbeispiel, auf das immer wieder verwiesen wird, ist der weihnachtliche Geschenkereigen. Genauer besehen ist jedoch unsere Privatsphäre insgesamt (die Ehe, die Familie, die Freundschaft) durchzogen von, wenn nicht gar gemacht aus Gabentauschbeziehungen. In vormodernen und insbesondere segmentären Gesellschaften spielt der Gabentausch demgegenüber eine in allen Sphären zentrale Rolle. Hierarchien sind wenig ausgeprägt, eine institutionalisierte politische Führung mit weitreichenden Steuerungskompetenzen gibt es nicht. Das Segment beziehungsweise die erweiterte Familie ist die basale politische und wirtschaftliche Einheit. Rein materiell sind die Segmente in der Regel autark. Frauen sind ihr wichtigstes

19 Maurice Godelier: L’Enigme du don, Paris 1996. 
>Kapital<, und Allianzen werden, im Rahmen einer weitgehend eingeschränkten Wahlfreiheit nicht nur der eigentlichen Partner, sondern ganzer Segmente auf dem Wege der Heirat geschmiedet. Der Frauentausch ist damit die wichtigste Institution segmentärer Gesellschaften. Die Religion ist kein präzise von sonstigen Überzeugungen und Praktiken geschiedener Bereich. Natur und Kultur, Diesseits und Jenseits werden, wenn nicht als Kontinuum, so jedenfalls als homologe, von Menschen wie Geistern und >übernatürlichen` Kräften besiedelte Bereiche aufgefasst. Man schuldet der lebensspendenden Natur Achtung, so wie umgekehrt diese auf die Gaben der Menschen angewiesen ist.

Die Reihe der Illustrationen für die Allgegenwart und das Gewicht des Gabentauschs in segmentären Gesellschaften ließe sich fortsetzen. Für den Begriff des Gabentauschs freilich kommt es auf seine formale Bestimmung an. Von Marcel Mauss stammt der Vorschlag, ihn, beziehungsweise die Gabe, einerseits durch die dreifache Verpflichtung zu geben, anzunehmen und zu erwidern, und andererseits in Spannung dazu durch die Ambivalenz, sowohl obligatorisch als auch freiwillig zu sein, zu definieren. ${ }^{20}$ Über Mauss' Erklärung oder Begründung dieser seltsamen Verpflichtung, die zugleich keine sein soll, sind Ströme von Tinte vergossen worden. Ich halte das Rätsel der Gabe (im Grunde schon von Mauss selbst) indes für weitgehend gelöst. ${ }^{21}$ Die Gabe ist der wirkliche, empirische Sozialvertrag segmentärer Gesellschaften, ein stets aufs neue bekräftigter Frieden, der ein komplexeres - etwa zweckrationales ${ }^{22}$

20 Siehe Marcel Mauss: »Die Gabe. Form und Funktion des Austauschs in archaischen Gesellschaften «, in: ders., Soziologie und Anthropologie, Bd. 2, Frankfurt/Main 1989, S. 9-144, hier S. 16f., 71-80.

21 Vgl. ebd., S. 140-142; Marshall Sahlins: »The Spirit of the Gift«, in: ders., Stone Age Economics, New York 1973, S. 149-183; Alain Caillé: Anthropologie du don. Le tiers paradgime, Paris 2000, S. 93-120.

22 Neben etlichen empirischen sprechen auch logische oder formale Einwände gegen die rational-choice-theoretische These vom Primat des seinfachen Tauschs. Sofern es den Zweck des einfachen Tauschs ausmacht, dass beide Tauschpartner sich tauschend besser stellen, muss das Tauschergebnis, das heißt der Zustand der Befriedigung nach Vollzug des Tauschs gedanklich und emotional schon vor Vollzug des Tausches vorweggenommen werden können. Nur sind weder die Präferenzen der Subjekte stabil noch die Resultate kalkulierbar, wenn zumindest einem der beiden Tauschpartner das Wissen um die sinnliche Qualität der Gegenleistung fehlt. Last not least ist es unter der Voraussetzung einer beidseits auf den eigenen Vorteil bedachten Berechnung fraglich, wie es überhaupt zum Tausch kommen kann. Jeder wird nämlich darauf schielen, sich der Güter des anderen möglichst ohne Gegenleistung zu bemächtigen, dem anderen >vernünftigerweise < jedoch genau dasselbe Kalkül unterstellen, so dass der vermeintlich spontane Austausch in Wahrheit stockt oder gar nicht erst in Gang kommt. Aufgelöst werden kann diese Situation doppelter Kontingenz durch Macht oder aber durch eine erste freiwillige, deswe- 
Handeln überhaupt erst ermöglicht, meine aber, dass die Rache darüber hinaus erhellt, worin dieser Frieden besteht und was mit ihm auf dem Spiel steht.

Rache, auch Blutrache, ist nicht nur nicht die Negation von Reziprozität, sondern auch nicht das potentielle Blutbad, als das sie häufig imaginiert wird. Richtig ist allerdings, dass gerade und vor allem der Mord an einem Clangenossen durch ein Gesellschaftsmitglied nach Rache schreit. (Andere typische Anlässe zur Rache sind Ehebruch, Vergewaltigungen, vorehelicher Sexualverkehr und Inzest. Eine weitere, wenn auch auf Grund der materiell beschränkten Standards segmentärer Gesellschaften sehr viel seltenere Kategorie sind Eigentumsdelikte.) Wer das Blut eines Clangenossen vergießt, vergießt gewissermaßen das Blut des Clans selbst. Das vergossene Blut ist Symbol für die nicht bloß physische, sondern auch psychosoziale Verletzung und Schwächung einer Gruppe. Es zählt nicht allein, dass ein Mord die betroffene Gruppe eines produktiven Mitglieds, eines Jägers, Kriegers oder Bauern, einer gebärfähigen Frau oder künftigen Braut beraubt, sondern genauso, dass die Integrität der Gruppe als ganzer, ihr Anspruch auf Unversehrtheit, verletzt wird. Selbstverständlich ist ein Mord ein Angriff auf das wirtschaftliche und biologische Reproduktionspotential eines Kollektivs. Es erstaunt deshalb nicht, dass es für die Gruppe des Mörders verbreitet die Möglichkeit gibt, den Mord durch die Gabe einer Frau zu kompensieren, die Heirat also ein Mittel sein kann, die Rache und erst recht Rachezirkel abzuwehren. Allerdings darf die Verletzung der Gruppe durch einen Mord nicht auf einen irgendwie objektivierbaren Schaden reduziert werden; immer auch steht zugleich mit dem Blut die Ehre, die Wehrhaftigkeit des Clans auf dem Spiel. Eben deshalb können Ehrverletzungen zu blutigen Auseinandersetzungen führen. La lessive de l'honneur ne se coule qu'au sang, heißt ein französisches Sprichwort. Blut und Ehre sind keine grundsätzlich geschiedenen, inkonvertiblen »Güter«; auch ist eine Kultur der Ehre, wie man sie etwa in weiten Teilen des Mittelmeerraums beobachten kann, ${ }^{23}$ kein Substitut für eine ältere und mittlerweile überwundene »Kultur des Blutes«, so als ob Ehrenhändel bloß eine zivilisiertere Form der Blutrache darstellten. Ehrkonflikte können, wofür ethnische Auseinandersetzungen oder der Kampf von Mafia-Banden Beispiele sind, weitaus blutiger sein als die gezielte Rache an Mördern und ihren

gen aber nicht interesselose Gabe. Der rationale Tausch wird faktisch möglich mithin erst auf der Grundlage eines scheinbar irrationalen Akts.

$23 \mathrm{Vgl}$. Jean G. Peristiany (Hg.): Honour and Shame. The Values of Mediterranean Society, Chicago 1966; Christian Giordano: »Der Ehrkomplex im Mittelmeerraum: sozialanthropologische Konstante oder Grundstruktur mediterraner Lebensform?«, in: Ludgera Vogt/Arnold Zingerle (Hg.), Ehre. Archaische Momente in der Moderne, Frankfurt/Main 1994, S. 172-192. 
Angehörigen. Was Blut und Ehre analytisch dennoch unterscheidet, ist, dass jenes ein Symbol darstellt eher für die zeitliche Einheit der Gruppe im Gefolge der Generationen, diese hingegen eine Chiffre vor allem für ihre soziale Autonomie im Geflecht der Allianzen.

Wenn die Rache also ein Anerkennungsverhältnis ist, dann nicht oder wenigstens nicht in erster Linie, insofern sie die Dauer der eigenen Gruppe durch eine der Verletzung korrespondierende Schädigung anderer Gruppen zu garantieren versucht, sondern dann deshalb, weil sie auf die Wiederherstellung eines Zustandes zielt, der durch die Gabe als zentraler Beziehung zwischen den Gruppen einer segmentären Gesellschaft allererst erzeugt wird. Einerseits setzt die Rache den Respekt zwischen den beteiligten Gruppen voraus, andererseits ist sie ein Reparaturmechanismus einer aktuell beschädigten Relation. Sie ist Vergeltung eines Torts, zugleich aber ein Plädoyer für die Rückkehr zum status quo ante. Die Kohäsion, der Zusammenhang und Zusammenhalt der Gesellschaft, wird durch einen legitimen Racheakt nicht aufs Spiel gesetzt, sondern praktisch beschworen. Es gibt die Pflicht, eine Ehrverletzung zu vergelten, nicht weil der Stolz einzelner keine Alternative zuließe, sondern weil die Ehre nur ein anderer Name ist für das rechte Maß von Nähe und Distanz zwischen den Segmenten einer Gesellschaft, aber auch für die Prekarität eines Zustands, der, wenn nicht der natürlichen Feindschaft der Menschen, so doch dem Misstrauen, das Fremdem gegenüber zunächst einmal angezeigt ist, nur auf dem Wege der Gabe mühsam abgetrotzt werden konnte.

Der Gabentausch ist die empirische Form des Sozialvertrags. Er ist kein Waren- oder Gütertausch, im Grunde nicht einmal die Übergabe irgendwelcher Gegenstände, sondern das Medium einer Relation wenigstens zweier Kollektive, die wechselseitig ihr prinzipielles Wohlwollen beteuern, sich als Gleiche anerkennen und gegebenenfalls verbünden. Eine Gabe wird nicht erwidert, um Schulden zu begleichen - denn die Unterbrechung oder gar Ablösung einer Beziehung ist gerade nicht intendiert -, sie wird erwidert, um sich einander der guten Absichten sowie des Beistands in schwierigen Zeiten zu versichern. Dieser Zustand ist nicht ein für alle Mal gegeben - es gibt kein Gesetz der Gabe, keine unverbrüchliche Garantie, das ein geschlossener Friede hält -, sondern er muss immer wieder angerufen und handelnd hergestellt werden. Die Verpflichtung, eine Gabe anzunehmen und zu erwidern, kann missachtet werden - das Gegenüber bleibt letztlich frei. Und dennoch bindet die Gabe, eben weil die Alternative die Wiederkehr des ursprünglichen Misstrauens ist. Dieses zu überwinden ist die Leistung der ersten Gabe. Sie ist keine Reaktion, sondern eine Offerte, eine Öffnung, ein riskanter Appell, überhaupt zu kooperieren. Noch gibt es nichts, keine Erfahrun- 
gen und keinen greifbaren Vorteil, welche eine positive Antwort nahe legten. Daher ihre Extravaganz, der Überschwang, der eine erste Gabe begleitet; ihr außerordentlicher Wert und die Großzügigkeit, die aus ihr spricht, sind die Provokation eines positiven Bescheids, ein In-Vorleistung-Treten, ein gewagter Versuch, der stets möglichen Aggression zuvorzukommen und sie zu bannen. Es ist diese Prekarität des Gabentauschs, seine der Freiheit der anderen geschuldete Kontingenz, die für seine Ritualisierung wie ideologische Überhöhung verantwortlich ist. Er ist ein Gut, eine zivilisatorische Errungenschaft ersten Ranges, die verletzt werden kann, aber nicht revidiert werden soll.

Und es ist dieses Gut, welches die >primitive< Rache letztendlich schützt. Sie ist bindend, sie verpflichtet zur Vergeltung, weil es sich bei Vergehen gegen die Ehre, gegen die Autonomie des Kollektivs nicht um lässliche Sünden, sondern um einen Angriff auf das Herzstück segmentärer Gesellschaften handelt. Aber sie ist auch penibel verregelt, um der Eskalation vorzubauen. Ohne Frage, Gabenzirkel müssen bekräftigt und unterhalten, Rachezirkel hingegen gezähmt und unterbrochen werden. ${ }^{24}$ Umgekehrt freilich läuft auch der Tausch von Gaben Gefahr, wie etwa im Falle des Potlatch überzogen zu werden und in offenen Kampf auszuarten, während die Rache die Integrität der Gesellschaft nur selten bedroht, sondern in der Regel vielmehr deren Bedrohung beklagt. Gabe und Rache sind Formen der Reziprozität; gleichwohl setzt diese jene voraus. Denn das Unrecht, das zur Rache berechtigt, suspendiert nicht die Ordnung, welcher der Gabentausch stiftet, sondern ist eine `Nahmeく, welche daran erinnert, dass die Ordnung zerbrechlich ist und deshalb gewahrt werden muss.

\section{IV.}

Die wilde Rache, welche unsere Phantasien beflügelt und uns zugleich so erschreckt, in der ein erstes Vergehen oder gar nur eine Fahrlässigkeit das Opfer oder seine Gefährten zur gewaltsamen Antwort nötigt, eine Rache, die eine Verletzung nur durch eine größere Wunde meint wettmachen zu können, in der Blut nur durch weiteres Blut vergolten werden kann und deshalb nur so strömt, ist, historisch betrachtet, keineswegs der Normalfall. Gleichwohl spricht aus solchen Vorstellungen mehr als nur der behagliche Schauer, der uns angesichts der vermeintlich barbarischen Praktiken der Wilden so leicht befällt und damit hilft, die eigene Zivilisa-

24 Siehe Wolfgang Eßbach: »Gabe und Rache. Zur Anthropologie der Gegenseitigkeit«, in: Gerburg Treusch-Dieter u.a. (Hg.), Schuld, Tübingen 1999, S. 11-20, hier S. 18. 
tion in ein vorteilhaftes Licht zu setzen. Denn auch wenn wilde Rache nicht oder nur selten die Rache der Wilden ist, ist sie keine bloße Phantasie, sondern sehr wohl ein Faktum, mehr als eine Aberration, der nur am Rande Rechnung zu tragen ist. Der springende Punkt jedoch ist ihre spezifische Modernität. Sie ist es, die mit dem Staatsbildungsprozess einherund aus ihm hervorgeht, der ihr eigentlich doch Einhalt gebieten soll, und die heute wiederkehrt unter den Bedingungen der Desintegration des staatlichen Gewaltmonopols.

Die traditionelle Rache darf weder mit Krieg noch mit Strafe verwechselt werden. Sie regelt vielmehr einen Zwischenbereich zwischen außergesellschaftlich-politischen und innerfamiliär-privaten Konflikten. Sie kann zu Rachekriegen auswachsen und als Strafmaßnahme instrumentalisiert werden, typisch jedoch werden diese Aus- und Überdehnungen erst, wenn ein anderen überlegener Herrscher beziehungsweise eine Zentralgewalt auf den Plan tritt, welcher oder welche die relative Gleichheit der Segmente durchbricht, sie aufhebt und das Prinzip der horizontalen Reziprozität in die Vertikale kippt. Richtig ist zwar, dass der werdende Staat grundsätzlich das Gewalt- und Jurisdiktionsmonopol, die Strafkompetenz wie das ius ad bellum für sich beansprucht und beanspruchen muss, falsch aber wäre es, daraus zu schließen, dass er seine Ansprüche immer auch hätte durchsetzen können und immer (schon) daran interessiert (gewesen) wäre, sie durch ein grundsätzliches Straf- und Gewaltverbot der Herrschaftsunterworfenen zu flankieren.

Die Staatsbildung war und ist ein kontingenter, voraussetzungsreicher und labiler Prozess, der nur in der westlichen Welt - und selbst dort nur unter Einsatz massiver Gewalt ${ }^{25}$ - in Form von nach innen pazifizierten und nach außen immerhin in ein Geflecht von kriegs- und völkerrechtlichen Selbstverpflichtungen eingebundenen Nationalstaaten seinem begrifflichen Ende zugeführt werden konnte. Seinen Zenit dürfte er indes schon längst überschritten haben. Außerhalb des Westens aber ist dieser Prozess entweder gar nicht erst angelaufen oder steckengeblieben, abgebrochen oder revidiert worden. ${ }^{26}$

Zunächst und heute wieder kommt es den Staaten darauf an, nur diejenige innergesellschaftliche Gewalt zu unterdrücken, die ihren unmittelbaren militärischen und wirtschaftlichen Interessen zuwiderläuft. Inwieweit Gesellschaftsmitglieder oder innergesellschaftliche Gruppen auf Gewalt zurückgreifen, um ihre internen Konflikte zu lösen, war und -

25 Siehe etwa Charles Tilly: »War Making as State Making «, in: Peter B. Evans u.a. (Hg.), Bringing the State Back In, Cambridge 1985, S. 169-191.

26 Vgl. Trutz v. Trotha: »Ordnungsformen der Gewalt oder Aussichten auf das Ende des staatlichen Gewaltmonopols«, in: Brigitta Nedelmann (Hg.), Politische Institutionen im Wandel (= Sonderheft 35 der Kölner Zeitschrift für Soziologie und Sozialpsychologie), Opladen 1995, S. 129-166. 
schaut man etwa auf die Duldung brutaler Bandenkriege in den Armutsgebieten unserer Großstädte - ist dabei häufig zweitrangig. Während einer Politisierung der Rache, die mit dem Entstehen von Staaten selbstverständlich weder als Diskurs noch als Praxis gewissermaßen auf Zuruf verschwindet, neue Hindernisse in den Weg gelegt werden, wird ihre Privatisierung und Individualisierung wenigstens strukturell durch die Zerstörung der segmentären Ordnung gefördert. Indem der Staat der Rache einerseits wesentliche ihrer traditionellen Anlässe, Aufgaben und Attribute entzieht, es andererseits allerdings unterlässt, ihre Akteure an gewaltsamem Handeln zu hindern, öffnet er ihrer >Deregulierung`Tür und Tor.

Und nicht nur das. Hinzu kommt, dass die Herrschaft sich auf ihrem Wege von der bloßen Machthaberschaft zur politischen Souveränität ihrerseits immer wieder auf das Prinzip der Vergeltung beruft. Die Zurschaustellung von Straftätern, vor allem aber ihre öffentliche und nicht selten spektakuläre Hinrichtung wird vordergründig als Akt dargestellt, welcher die Rachegelüste des Volkes befriedigt, faktisch inszeniert jedoch als Apotheose der Majestät und deren Unantastbarkeit. ${ }^{27}$ Hier wird kein Gleichgewicht wiederhergestellt, sondern Herrschaft verabsolutiert. Ebenso gab und gibt es unzählige Fälle, in denen die Machthaber einen bereits etablierten Staatsapparat für die Bereicherung, den Machterhalt persönliche Rachefeldzüge instrumentalisieren. In nicht wenigen Staaten operieren Polizei und Justiz, eigentlich damit betraut, als neutrale Dritte über die Einhaltung der Gesetze zu wachen, vornehmlich oder allein im Dienste einer herrschenden Clique. Eine formal legale, tatsächlich aber despotische Herrschaft delegitimiert die dritte Gewalt und leistet auf diese Weise wahrhaft wilden Formen der Selbsthilfe Vorschub. Das Recht wird zur Hülse, zur Beute der Staat.

Der Privatisierung und Individualisierung der Rache folgt ihre Vermarktung meist auf dem Fuße. Es entstehen »Gewaltmärkte«, deren Akteure sich zwar noch - oder wieder - auf buchstäblich ehrenwerte Motive wie das Recht und die Pflicht zur Rache berufen, die Machtmittel, über die sie verfügen, in Wahrheit jedoch kalt kalkuliert investieren oder meistbietend verkaufen. ${ }^{28}$ Die Rache wird in den Dienst wirtschaftlicher Zwecke gestellt. Keine Tradition wacht mehr über ihre Gegenstände und über ihr Maß. Das muss die Gewalt nicht intensivieren - schließlich ist diese selbst eine Ressource, die gehegt und gepflegt oder gekauft werden muss -, kann aber zu einer Brutalisierung der Verhältnisse führen, wenn

27 Siehe Michel Foucault: Überwachen und Strafen. Die Geburt des Gefängnisses, Frankfurt/Main 1977, S. 44-90.

28 Vgl. Georg Elwert: »Gewaltmärkte. Beobachtungen zur Zweckrationalität von Gewalt«, in: Trutz v. Trotha (Hg.), Soziologie der Gewalt (= Sonderheft 37 der Kölner Zeitschrift für Soziologie und Sozialpsychologie), Wiesbaden 1997, S. 86-101. 
Gewaltunternehmer ihr Geschäft mit dem Diskurs der Rache bemänteln. ${ }^{29}$ Aus der Rache als einem Instrument, seine und seiner Gruppe Ehre zu verteidigen, wird dann ein Mittel des Gelderwerbs, der seinerseits vielleicht zwar eine Art Ehre des Geldes abwirft, an sich aber keinerlei innere Grenzen kennt. Gewiss, die Ehre war immer schon mehr als nur ein qua Geburt erworbener Rang, der Anspruch zumal, die Ehre seines Standes nicht nur nicht zu enttäuschen, sondern möglichst zu mehren, mit ihrer Monetarisierung jedoch wird sowohl ihre Subjektivierung wie objektiv die Konkurrenz um dieses symbolische Kapital auf die Spitze getrieben.

Ja, die Rache kehrt wieder, aber es ist nicht die Rache der Wilden, welche uns heimsucht. Die Rache, die uns heute umbrandet, als die uns etwa die Hinrichtung Bergs verkauft werden soll, ist das Produkt einer Welt, in welcher Staaten formal zwar allerorten regieren, faktisch aber an immer neuen Stellen den Rückzug antreten, einer Welt, in der eine entfesselte Ökonomie die Restbestände von Tradition pervertiert. Die Rache wird zum oder ist bereits Vorwand zur schamlosen Bereicherung und Beseitigung der Konkurrenz. Man beruft sich auf sie, um die Gewalt zu kaschieren, mit der man private Ziele verfolgt. Politisch wird sie zum Mittel der Kriegsführung, zur heimtückischen Waffe in der Hand derer, welche ihre Gegner moralisch und rechtlich nicht als Gleiche behandeln. Vorausgesetzt werden keine gemeinsamen Werte, betont wird die ideologische Kluft, welche die Feinde trennt. Zweck eines Racheakts wie der Hinrichtung Bergs ist nicht die Regulation, nicht die Lösung eines Konflikts, sondern dessen Verschärfung. Gleichwohl zehren die Täter, die ihr Tun als Rache bezeichnen, von einer Art >Legitimationsüberschuss〈. Wer Rache sagt, fühlt sich im Recht, will zumindest den Anschein erwecken, im Recht zu sein. Genauer gesagt, Recht wird beansprucht, ihm unterwerfen aber will man sich nicht. Die (mediale Welt-)Öffentlichkeit wurde von Bergs Henkern nicht angerufen, um ein Urteil über die Legitimität ihres >Kampfes〈zu sprechen, dem man sich gegebenenfalls beugte, sondern um Terror zu säen und Reklame zu machen für eine moralisch vorab entschiedene Sache. $\mathrm{Zu}$ hoffen bleibt, dass jene grausamen Bilder das Gegenteil dessen bewirken, was sie bezwecken - ebenso freilich, dass uns angesichts der Misshandlungen von Abu Ghraib der Ekel befällt. Denn was hier verspielt wurde, ist die Ehre des Westens.

29 Vgl. Stephanie Schwander-Sievers: "Wer besitzt die >Lizenz zum Töten< in Albanien? oder: Fragen zu Gruppensolidarität und Gewaltlegitimation in einer sanderen Modernisierung«", in: Jan Koehler/Sonja Heyer (Hg.), Anthropologie der Gewalt. Chancen und Grenzen der sozialwissenschaftlichen Forschung, Berlin 1998, S. 71-88. 


\section{Literaturverzeichnis}

Bohannan, Paul (Hg.): Law and Warfare. Studies in the Anthropology of Conflict, Austin, London 1967.

Caillé, Alain: Anthropologie du don. Le tiers paradgime, Paris 2000.

Elster, Jon: »Norms of Revenge«, in: Ethics 100 (1990), S. 862-885.

Elwert, Georg: »Gewaltmärkte. Beobachtungen zur Zweckrationalität von Gewalt «, in: Trutz v. Trotha (Hg.), Soziologie der Gewalt (= Sonderheft 37 der Kölner Zeitschrift für Soziologie und Sozialpsychologie), Wiesbaden 1997, S. 86-101.

Eßbach, Wolfgang: »Gabe und Rache. Zur Anthropologie der Gegenseitigkeit«, in: Gerburg Treusch-Dieter u.a. (Hg.), Schuld, Tübingen 1999, S. 11-20.

Esser, Hartmut: Soziologie. Spezielle Grundlagen, Bd. 3: Soziales Handeln, Frankfurt/Main, New York 2000.

Evans-Pritchard, Edward E.: »The Nuer of the Southern Sudan«, in: Meyer Fortes/Edward E. Evans-Pritchard (Hg.), African Political Systems, London 1940, S. 272-296.

Filkins, Dexter: »Iraq Videotape Shows the Decapitation of an American«, in: New York Times vom 12.05.2004.

Foucault, Michel: Überwachen und Strafen. Die Geburt des Gefängnisses, Frankfurt/Main 1977.

Giordano, Christian: »Der Ehrkomplex im Mittelmeerraum: sozialanthropologische Konstante oder Grundstruktur mediterraner Lebensform?«, in: Ludgera Vogt/Arnold Zingerle (Hg.), Ehre. Archaische Momente in der Moderne, Frankfurt/Main 1994, S. 172-192.

Girard, René: Das Heilige und die Gewalt, Zürich 1987.

Godelier, Maurice: L'Enigme du don, Paris 1996.

Gould, Roger V.: »Revenge as Sanction and Solidarity Display: An Analysis of Vendettas in Nineteenth-Century Corsica«, in: American Sociological Review 65 (2000), S. 682-704.

Hoebel, E. Adamson: »Feud: Concept, Reality and Method in the Study of Primitive Law«, in: Akshayakumar R. Desai (Hg.), Essays on Modernization of Underdeveloped Societies, Bd. 1, Atlantic Highlands, N.J. 1976, S. 500-513.

Jacoby, Susan: Wild Justice. The Evolution of Revenge, New York 1983.

Jonassohn, Kurt: Genocide and Gross Human Rights Violations in Comparative Perspective, New Brunswick, N.J. 1998.

Kelsen, Hans: Vergeltung und Kausalität, Wien 1982.

Koch, Klaus-Friedrich: War and Peace in Jalémó. The Management of Conflict in Highland New Guinea, Cambridge, Mass. 1974.

Malinowski, Bronislaw: Crime and Custom in Savage Society, London 1926. 
Mauss, Marcel: »Die Gabe. Form und Funktion des Austauschs in archaischen Gesellschaften«, in: ders., Soziologie und Anthropologie, Bd. 2, Frankfurt/Main 1989, S. 9-144.

Peristiany, Jean G. (Hg.): Honour and Shame. The Values of Mediterranean Society, Chicago 1966.

Rieder, Jonathan: »The Social Organization of Vengeance«, in: Donald Black (Hg.), Toward a General Theory of Social Control, Bd. 1: Fundamentals, Orlando 1984, S. 131-162.

Sahlins, Marshall: »The Spirit of the Gift«, in: ders., Stone Age Economics, New York 1973, S. 149-183.

Scheler, Max: Das Ressentiment im Aufbau der Moralen, Frankfurt/Main 1978.

Schwander-Sievers, Stephanie: »Wer besitzt die >Lizenz zum Töten< in Albanien? oder: Fragen zu Gruppensolidarität und Gewaltlegitimation in einer sanderen Modernisierung « in: Jan Koehler/Sonja Heyer (Hg.), Anthropologie der Gewalt. Chancen und Grenzen der sozialwissenschaftlichen Forschung, Berlin 1998, S. 71-88.

Steinmetz, Sebald R.: Ethnologische Studien zur ersten Entwicklung der Strafe. Nebst einer psychologischen Abhandlung über Grausamkeit und Rachsucht, 2 Bde., 2. Aufl., Groningen 1928.

Thurnwald, Richard: »Art. >Blutrache««, in: Reallexikon der Vorgeschichte, Bd. 2, Berlin 1925, S. 30-41.

Tilly, Charles: »War Making as State Making «, in: Peter B. Evans u.a. (Hg.), Bringing the State Back In, Cambridge 1985, S. 169-191.

Trotha, Trotz v.: »Ordnungsformen der Gewalt oder Aussichten auf das Ende des staatlichen Gewaltmonopols«, in: Brigitta Nedelmann (Hg.), Politische Institutionen im Wandel (= Sonderheft 35 der Kölner Zeitschrift für Soziologie und Sozialpsychologie), Opladen 1995, S. 129-166.

Verdier, Raymond: »Le Système vindicatoire. Esquisse théorique «, in: ders. (Hg.), La Vengeance. Etudes d'ethnologie, d'histoire et de philosophie, Bd. 1: Vengeance et pouvoir dans quelques sociétés extra-occidentales, Paris 1981, S. 11-42. 
Bereitgestellt von | Universitaetsbibliothek Basel

Angemeldet

Heruntergeladen am | 04.10.18 17:47 